Écrire

l'histoire

\section{Écrire l'histoire}

Histoire, Littérature, Esthétique

3| 2009

Le détail (1)

\title{
Le savant et le bouton de culotte
}

Érudition et roman historique dans La Semaine sainte

\section{Nathalie Piégay-Gros}

\section{OpenEdition}

Journals

Édition électronique

URL : http://journals.openedition.org/elh/936

DOI : $10.4000 /$ elh.936

ISSN : 2492-7457

Éditeur

CNRS Éditions

\section{Édition imprimée}

Date de publication : 1 juin 2009

Pagination : 35-43

ISBN : 978-2-35698-008-3

ISSN : 1967-7499

Référence électronique

Nathalie Piégay-Gros, «Le savant et le bouton de culotte », Écrire l'histoire [En ligne], 3 | 2009, mis en ligne le 01 juin 2012, consulté le 23 septembre 2020. URL : http://journals.openedition.org/elh/936 ; DOI : https://doi.org/10.4000/elh.936 


\section{Le savant et le bouton de culotte Érudition et roman historique dans La Semaine sainte}

文 Dominique Arban, en 1968, Aragon explique qu'il a dû abandonner un roman qui se situait en plein XII ${ }^{\mathrm{e}}$ siècle, sous Louis VIII, et qu'il avait alors considéré « le roman historique comme une impossibilité matérielle ${ }^{1}{ }$. C'est que le roman, à la différence du poème, qui, lui, « permet d'aller beaucoup plus vite d'un point à un autre d'une époque », et dans lequel « le bouton de culotte ne joue pas le rôle principal ${ }^{2}$ », exige une précision dans la reconstitution historique et un savoir détaillé. Le roman ne peut donc pas prendre la tangente ni emprunter des raccourcis qui fassent l'économie du monde concret: «ne pas savoir comment s'attachaient alors les vêtements, ne pas avoir une représentation exacte du bouton au XII ${ }^{\mathrm{e}}$ siècle, devoir étudier l'exact état du tissage à une époque, la nature des étoffes, pour m'en tenir à l'exemple de l'habillement, sans parler de l'état des routes et de la technique de leur construction, c'était déjà pour un réaliste une raison suffisante d'échec », écrit encore Aragon en $1959^{3}$. La question de la représentation concrète et précise de la réalité dessine donc une ligne de partage assez nette entre le poème et le roman, qui l'un et l'autre, il est important de le souligner, peuvent être historiques et réalistes pour Aragon lecteur de Hugo, bien qu'ils ne mettent pas en œuvre les mêmes moyens pour représenter le monde réel. La Semaine sainte, roman historique, quoi qu'en dise son auteur, peut être lu comme une revanche prise sur le roman médiéval échoué et abandonné en 1947. Les connaissances sur l'état exact du tissage, sur les outils, la nature des étoffes, les routes et les moyens de déplacement, mais aussi les documents permettant de préciser les conditions météorologiques et de spécifier quels furent les acteurs, principaux ou secondaires, de la fuite du roi Louis XVIII, pendant la semaine sainte de

1. Louis Aragon, Dominique Arban, Aragon parle avec Dominique Arban, Paris, Seghers, 1968, p. 155.

2. Louis Aragon, Entretiens avec Francis Crémieux, Paris, Gallimard, 1964, p. 32.

3. Louis Aragon, J'abats mon jeu, Paris, Éditeurs français réunis, 1959, p. 154. 
1815, sont suffisamment nombreux pour écrire un roman qui compte plus de cinq cents pages en collection «Blanche » chez Gallimard, alors que sa diégèse ne compte, elle, qu'une semaine amputée de son septième jour.

L'érudition mise en œuvre pour écrire ce roman est en effet d'une ampleur considérable: les documents qu'a lus Aragon lui ont livré une masse de détails qu'il a explorée en romancier. Bien plus qu'une vue panoramique sur les Cent-Jours, qui débutent avec cette semaine sainte de 1815, Aragon cherche dans les récits qu'il se procure et les documents qu'il rassemble des précisions relatives aux realia - état des routes, monde du travail, costumes, etc. - et au destin des personnages historiques. C'est ainsi qu'il se documente sur la manufacture textile de Van Robais ou encore sur la manufacture de Klingenthal: les termes techniques relatifs aux lames de cavalerie (p. $942{ }^{4}$ ) sont issus du rapport sur les « Procédés de fabrications des lames de sabres à la manufacture de Klingenthal » du capitaine Hervilleo, consulté par Aragon. L'érudition donne donc lieu à des détails dont la précision se lit dans le lexique technique: le «touloup » (p. 1083) que porte Simon Richard, le «bouzin » (p. 1019) que l'on extrait avec la tourbe, la «vache », ce soufflet que l'on manie dans la forge (p. 930), sont autant de termes issus de documents historiques qui confor- tent la représentation réaliste et l'orientent vers une forme de didactisme.

Les personnages sont l'objet d'une représentation particulièrement détaillée qui s'enracine dans des lectures érudites tout entières dirigées par les nécessités de l'invention romanesque. Même les personnages secondaires et les figures qui apparaissent de manière très fugace, si nombreux dans ce roman qui n'est pas seulement celui du peintre Géricault, ont été l'objet d'une enquête très minutieuse, comme en témoignent par exemple les tentatives pour en savoir plus sur la famille Degeorge ou sur Descrivieux, personnage découvert dans les Confidences de Lamartine (p. 1218). Cependant, si l'exactitude historique et la précision quasi maniaque de la représentation, souvent remarquées par les historiens qui s'intéressent aux Cent-Jours ou à Géricault, procèdent de lectures nombreuses, sérieuses, celles-ci restent cavalières. Aragon lit beaucoup, mais de manière très ciblée: les pages découpées dans les livres conservés au moulin de Saint-Arnoult sont souvent celles qui concernent la seule semaine sainte de 1815, sans poursuivre hors des limites de la diégèse les mémoires et biographies. Car ce sont essentiellement des textes de ce type qu'a lus Aragon pour La Semaine sainte: les mémoires écrits sous la Restauration par les acteurs de l'histoire qu'il raconte - Lamartine, Chateaubriand, mais

4. Toutes les références renvoient à notre édition de La Semaine sainte dans Louis Aragon, Euvres romanesques complètes, Paris, Gallimard (Bibliothèque de la Pléiade), tome IV, 2008. 
aussi Macdonald, Marmont, Toustain, etc. -, les biographies des personnages - Géricault au premier plan, mais aussi Marmont, Fabvier, Berthier, le duc de Berry (par André Castelot...), le conspirateur Didier, etc. - nourrissent l'œuvre en détails romanesques dont l'origine est donc historique et livresque. Les anecdotes sont nombreuses, qui donnent à la fiction une épaisseur et une saveur certaines : le lavage des pieds des pauvres, le jeudi saint, par Louis XVIII, par exemple, rapporté par Macdonald et par Tony de Reiset dans leurs mémoires; le nom de la représentation donnée à Béthune, La Partie de chasse, au cours de laquelle des marques indubitables de dévouement au roi sont données; ou encore les lettres adressées par Berthier à $\mathrm{M}^{\text {me }}$ Visconti, sa maîtresse, interceptées par les Russes pendant la campagne de 1812, et qui firent scandale par la précision de leurs propos galants... (p. 1066). Aragon n'invente donc pas nombre de détails qui peuvent sembler, à la lecture, fictionnels (le nom de la bonne d'enfants de Berthier, M ${ }^{\text {elle }}$ Gallien, née à Tournus, par exemple, procède de la biographie écrite par Derrécagaix, que démarque précisément Aragon pour raconter la mort du maréchal à Bamberg). Le romancier fait donc preuve d'un scrupule historique tel que le tissu de la fiction s'apparente à un ensemble de citations que rien, la plupart du temps, ne vient expliciter.
Certains détails n'ont aucune valeur fonctionnelle : ils ne caractérisent pas, d'un point de vue politique, les protagonistes, contrairement aux deux premières anecdotes que nous venons de citer, ni ne servent la construction de personnages, comme le fait la troisième, relative au grand amour de Berthier pour $\mathrm{M}^{\mathrm{me}}$ Visconti. Que le $3^{\mathrm{e}}$ escadron du $1^{\text {er }}$ de chasseurs ait à sa tête le commandant Lanthonnet (p. 898), que le colonel Simonneau, qui remplace Saint-Chamans, ait « un accent de l'Hérault du tonnerre de Dieu» (p. 897), sont autant de détails issus de la documentation historique consultée par Aragon (en particulier les mémoires de Saint-Chamans, qui lui donnent les noms de tous les comparses qu'on retrouve dans ce chapitre; voir note p. 896), mais ils n’ont pas de fonction précise dans le texte. De même, lorsque est évoquée la vie de Marmont, le fait que son épouse soit une «fille de finances » (p. 876), détail rapporté par Pierre de Saint-Marc dans sa biographie du maréchal parue chez Fayard en 1957, relève de la caractérisation incidente d'un personnage en tous points secondaire. Ces détails ont donc pour finalité principale d'assurer la $c r e ́-$ dibilité de la représentation historique et ajoutent au réalisme ${ }^{5}$.

L'érudition dont fait preuve ce roman assure bien une forme de revanche sur l'impossibilité du roman historique médiéval, pour lequel Aragon

5. À propos du vers de Victor Hugo: «Il avait dans la poche une toupie en buis » (« Souvenir de la nuit du 4 »), Aragon note que « le détail fait croire au tout » et qu'il s'agit là du « détail typique du réaliste »; Louis Aragon, «La Nuit du 4 », dans Euvres poétiques complètes, Paris, Gallimard (Bibliothèque de la Pléiade), tome II, 2007, p. 1346. 
semble n'avoir pas pu se représenter concrètement, de manière détaillée, la réalité qu'il voulait évoquer. Le remarquable, dans La Semaine sainte, est que cette érudition soit toujours orientée vers des fins romanesques, au point que son statut livresque soit très vite oublié, sauf peut-être lorsque le réalisme se fait didactique, comme dans les scènes de «La Nuit des arbrisseaux » et de « La Vallée de la Somme ». Les procédés d'inclusion du savoir historique dans le texte du roman sont si variés que le risque d'un collage brut et didactique de la documentation est le plus souvent évité et que l'origine érudite des détails n'apparaît pas dans la fiction. Autant il est évident que les personnages et les grandes étapes de l'intrigue sont historiques, et reposent donc sur une documentation en amont du romancier, autant les détails peuvent paraître fictionnels. Or, le plus souvent, il n'en est rien. Ainsi, pour raconter l'arrivée du roi à Béthune, Aragon emprunte à L'Histoire de Béthune de l'abbé Cornet nombre de détails qui pourraient sembler inventés : le nom des comparses (sœur Félicité, M ${ }^{\text {me }}$ Brassart; p. 16321633), celui du maire, M. Delalleau, du lieutenant Huet (p. 990), etc. Il amplifie et récrit la scène sur un mode burlesque, en renchérissant sur les détails fournis. Un aperçu est donné du physique et du passé de sœur Félicité:

La première personne qui se rendit compte de ce qui se passait fut une vieille femme encapuchonnée de noir, une ancienne religieuse de l'hôpital Saint-Jean, rentrée dans le siècle pendant la Révolution, par permission épiscopale, sœur Félicité. (p. 989)
Les emprunts à l'abbé Cornet n'interdisent donc pas des détails qui font de $\mathbf{M}^{\text {me }}$ Brassart un personnage certes secondaire, mais romanesque: elle est « en robe de chambre et bigoudis », c'est « la femme du marchand de fer» (p. 989). Seul le roman peut faire ainsi surgir des silhouettes concrètes et ouvrir des perspectives fugaces sur leur vie et leur histoire. C'est une pratique fréquente dans La Semaine sainte: non content de reprendre les informations précises fournies par les récits qu'il lit pour écrire son roman, Aragon en ajoute d'autres. Ainsi, à propos de Macdonald, il note que son père était jacobite, information qui lui est fournie par les Souvenirs du maréchal, mais invente que Macdonald était violoniste (p. 733). Que ce maréchal d'Empire lise du Haydn le dimanche des Rameaux, pour oublier ses rhumatismes, ouvre soudain une perspective sur son intimité et sa subjectivité, qui, paradoxalement, n'apparaissent que peu dans ses Mémoires. Un détail fictionnel et romanesque se greffe donc sur un détail historique: la récurrence de cette forme d'amplification montre que la lecture des documents nécessaires à la représentation est un tremplin pour l'invention. Car Aragon ne s'assujettit jamais au texte qui lui fournit les détails historiques dont il a besoin mais les oriente toujours vers les nécessités de la fiction. Ainsi, il peut ne pas reprendre un détail pourtant mentionné par la source qu'il utilise si le point de vue narratif suppose qu'il est ignoré du personnage. 
Dans le chapitre « Le Printemps », pour lequel les Mémoires de Saint-Chamans fournissent l'essentiel de l'information, il ne mentionne pas le nom du militaire auprès duquel le colonel Simonneau, ne sachant pas s'il faut aller à Saint-Denis ou se diriger sur Fontainebleau, part se renseigner,

avec son menton rond, dans le soleil matinal. Chez ce général qui leur avait fait la veille un petit discours de derrière les fagots, sur la place Louis-XV. Paraît que c'est un Autrichien, qu'il s'est battu contre nous à Essling, et qu'il n'a pris le service de la France qu'en 1811. (p. 900)

Ce général, dont le nom est ignoré par celui qui focalise la scène et la raconte, est Roussel d'Hurbal, que mentionnent les Mémoires de SaintChamans. Aragon en tait le nom, mais reprend les informations qui apparaissent dans les notes des Mémoires (la bataille d'Essling entre autres), comme si l'information lue ne pouvait rester complètement à l'écart de la fiction. Gette scène avait déjà été évoquée dans un des premiers chapitres du roman, «Quatre vues de Paris », selon le même point de vue, par les chasseurs irrités de l'attente imposée à leur régiment:

Qui était ce général qui les avait affligés d’un laïus des plus ridicules? On s'était bien tenu, on n'avait pas fait de réflexions, mais là-dessus, cette idée qu'il avait eue, le colonel, de signifier aux capitaines Riquet et Bouvard qu'ils avaient à rentrer à Béthune, au dépôt! (p. 719)
Dans ses Mémoires, Saint-Chamans écrit qu'il « harangua en peu de mots » les officiers rassemblés le 19 au matin sur la place Louis-XV, «pour leur présenter leurs devoirs envers le roi, dont ils n'avaient jamais reçu que des bontés ». Le détail volontairement tu est, comme celui qui est précisé, un facteur de la crédibilité romanesque: il permet en l'occurrence de renforcer l'impression de désordre et d'improvisation qui caractérise les premiers moments des Cent-Jours. Aragon, s'il suit scrupuleusement l'information factuelle fournie par les Mémoires, inverse très souvent la valeur qui lui est accordée, en particulier dans les scènes qui sont focalisées par les soldats de Napoléon. C'est le cas, par exemple, dans tout le chapitre « Le Printemps » : l'amplification de certains détails fournis par les mémoires de Saint-Chamans se retourne, dans le roman, contre leur auteur. On lit ainsi dans les Mémoires:

Le 21 mars au matin, je notifiai donc hautement l'intention où j'étais de quitter le commandement de ce régiment, et je chargeai un officier, assez bon maquignon, de faire vendre mes chevaux, qui ne m'étaient plus bons à rien et me devenaient à charge. ${ }^{6}$

Aragon brode sur cette trame, amplifie certains éléments et ajoute un détail (un «bai miroité », en parfaite cohérence avec l'attention minutieuse portée, dans toute La Semaine sainte, aux chevaux) :

6. Mémoires du général Comte de Saint-Chamans, ancien aide-de-camp du maréchal Soult, 1802-1832, Paris, Plon et Nourrit, 1896, p. 348. 
Pour un homme embêté, c'était un homme embêté: il y avait vingt-quatre heures que ses officiers le faisaient tourner en bourrique, il lui avait fallu toute la nuit pour se décider à quitter son régiment. On avait raconté à Simonneau que le Saint-Chamans, comme premier geste pour marquer le caractère irrévocable de sa résolution, avait fait venir un de ses capitaines et l'avait prié de se charger de vendre ses chevaux. Un officier qui avait fait toutes les guerres de l'Empire! À quoi il pense quand Napoléon revient, à vendre ses chevaux! Simonneau, pour sa part, aurait bien racheté le bai miroité de son prédécesseur, mais cela aurait fait jaser, sans parler du prix. Et puis, il était un peu trop fin, le miroité. Autant garder son alezan, lourd, mais résistant. (p. 896)

L'historicité du détail, dans La Semaine sainte, tient donc à l'origine de la documentation - mémoires, biographies, documents d'archives...autant qu'aux différents procédés de motivation mis en œuvre par le romancier. L'ampleur des emprunts est telle que La Semaine sainte peut être lu comme un roman-centon. Le roman historique réaliste se construit comme une mosaïque de fragments textuels que la fiction assimile sans les démarquer ${ }^{7}$. La force d'assimilation, l'impérialisme de la fiction sont alors remarquables et les procédés qui consistent à implanter dans le roman les fragments de lecture très efficaces. La rencontre de Géricault et d'Augustin Thierry, qu'Aragon invente, donne lieu ainsi à un assez long dévelop- pement du peintre sur Le Caravage, en lequel il voit un précurseur d'une forme de réalisme qu'il fera sienne:

On l'a blâmé de son naturel chagrin: c'est lui, disaiton, qui le pousse à charger personnages et objets de leurs ombres, il les éclaire à peine, et de haut, et le goût des oppositions fait que ses fonds sont obscurs, que ses bonshommes sont sans nuances, sans dégradé, et semblent posés sur un seul plan, sur un écran de ténèbres. L'abbé Lanzi dit que ses figures habitent une prison, qu'il n'y a chez lui ni correction du dessin, ni choix de cette beauté... Ah, parce que la vérité lui suffisait, qu'il n'avait pas soin de l'embellir, d'un arrangement de draperie, d'un truc imité d'une statue grecque! C'est bien noir, lui disait-on, la vie n'est pas ainsi... (p. 761)

\section{C'est bien à l'abbé Lanzi et à son Histoire de la} peinture en Italie que se réfère ici Aragon, qui dissémine dans le discours de Géricault des citations précises de l'historien:

[...] guidé ensuite par son naturel sombre et mélancolique, il [Le Caravage] se mit à représenter les objets très-peu éclairés et fortement chargés d'ombres. Il semble que ses figures habitent une prison, où elles ne reçoivent qu'une faible lumière par le haut. C'est ce qui fait que ses fonds sont toujours ténébreux, que ses personnages posent sur un seul plan, et qu'il n'observe aucune dégradation dans ses peintures, et cependant elles charment le spectateur par le grand effet qui résulte de ce contraste d'ombre et de lumière. Il ne faut chercher en lui, ni correction de dessin, ni choix de beauté. Il ne faisait aucun cas des études auxquelles

7. Philippe Hamon a bien noté cette tendance du roman réaliste à procéder par juxtaposition de fragments et à donner l'impression d'un « émiettement synecdochique »; Philippe Hamon, "Un discours contraint », dans Littérature et réalité, Paris, Seuil (Points), 1982, p. 162. 
s'arrêtaient les autres peintres pour ennoblir un[e] expression de tête ou pour représenter une belle draperie, ou imiter une statue grecque. Le beau n'était pour lui que le vrai quel qu'il fût. ${ }^{8}$

La paraphrase est un facteur efficace de précision et d'historicité: Géricault interprète Le Caravage en homme de son temps, comme pouvaient le faire ses contemporains. L'historicité du détail est évidente non seulement dans la représentation du réel, mais aussi dans son interprétation.

Pour autant, la prolifération de détails fait courir à l'écriture le risque d'une diffraction excessive. L'émeute de détails qui assaille l'esprit de l'artiste « accoutumé à exercer surtout sa mémoire et son imagination » conduit inévitablement à une forme d'anarchie, lorsque nulle hiérarchie forte ne gouverne plus la forme. Risque de dysharmonie donc, selon Baudelaire, qui voit dans la tyrannie des détails, «qui tous demandent justice avec la furie d'une foule amoureuse d'égalité absolue », une conséquence de l'esprit démocratique ${ }^{9}$. Dans La Semaine sainte, l'inflation du détail, qu'il ne s'agit pas d'interpréter selon les termes de la politique de l'esthétique baudelairienne, correspond néanmoins à la représentation d'un moment de crise politique exceptionnelle, où le désordre, l'improvisation, l'anarchie dominent. Sans doute est-ce une des raisons pour lesquelles l'écriture du détail menace parfois la cohérence de la représentation réaliste. Le centon est donc profondément ambivalent: d'un côté, il renvoie à la passion méticuleuse d'une écriture soucieuse d'historicité au point de faire de la fiction un montage de fragments textuels censés dire le réel avec la plus grande exactitude; de l'autre, il tend vers l'éparpillement, la diffraction, l'impossibilité de la représentation. On semble parfois perdre de vue, en effet, ce caractère dénombrable et dénommable du réel ${ }^{10}$ qui est le propre du réalisme lorsque $\mathrm{La}$ Semaine sainte cède à la pente vertigineuse d'une défense de l'infini: l'infini des détails et des noms qui les disent. Les paradigmes de patronymes déclinés de manière purement jubilatoire font courir à la représentation le risque d'une fracture certaine. Le détail n'est plus au service de la cohérence du récit, cet autre nom du réalisme pour Aragon, mais tourne en roue libre. Ce risque est perceptible dès le premier chapitre du roman, où la diffraction des noms des personnages en titres, surnoms, prénoms divers, fait parfois oublier leur entité et compromet la force de la représentation. Le roman nous plonge d'em-

8. Abbé Lanzi, L'Histoire de la peinture en Italie, depuis la renaissance des beaux-arts, jusques vers la fin du XVIII ${ }^{e}$ iècle, Paris, Seguin et Dufart, 1824, tome II, p. 195.

9. Charles Baudelaire, «Le peintre de la vie moderne », dans Euvres, Paris, Gallimard (Bibliothèque de la Pléiade), tome II, 1976, p. 699.

10. «Le monde est descriptible, accessible à la dénomination »; si le réel y est « envisagé comme un champ complexe et foisonnant, discontinu, "riche" et nombrable, dénommable », c'est qu'il s'agit bien « d'en faire l'inventaire »; Philippe Hamon, «Un discours contraint », art. cit., p. 162. 
blée dans l'épaisseur du monde, semblant d'abord renoncer à la vision surplombante garante d'une compréhension du réel qui devrait être pour Aragon la finalité du roman historique, à ce panora$m a$ qui est pour lui l'autre nom du roman. Le détail est alors coupé de la crédibilité qu'il doit apporter à la représentation. Il ne fait plus illusion.

Dès l'époque surréaliste, celle de La Défense de l'infini, Aragon avait noté cette tendance du détail à l'autonomisation propice à la « concentration émotive » :

Les enfants, poètes sans être artistes, fixent parfois un objet jusqu'à ce que l'attention le grandisse, le grandisse tant, qu'il occupe tout leur champ visuel, prend un aspect mystérieux et perd toute corrélation avec une fin quelconque. Ou ils répètent inlassablement un mot, tant et si bien qu'il se dépouille de tout sens pour demeurer un vocable poignant et sans but, qui parvient à leur tirer les larmes. ${ }^{11}$

Cette « concentration émotive » correspond à la part de l'affect propre à l'effet de réel qu'ont notée Barthes et à sa suite Michel Charles ${ }^{12}$. Part de l'affect en ce sens que le détail est ce qui excède et défie la représentation, mais aussi donne à lire la dimension poétique et fantasmatique du regard porté sur la réalité. Elle est particulièrement sensible lorsque sont évoqués les corps des militaires, les costumes des femmes, les uniformes chatoyants...
L'inflation du détail, dans ce roman, traduit donc le souci historique et réaliste d'Aragon. Mais la « cohérence du récit » réaliste est doublement menacée: d'une part, par une tendance métonymique à l'émiettement, à la diffraction; par une force centrifuge, d'autre part, force de transport métaphorique, qui fait courir le risque de l'éclatement. Toutes deux menacent la cohésion de l'écriture et la cohérence de la représentation, la première en ce qu'elle affiche le caractère discontinu des représentations à l'origine de la fiction, la seconde en ce qu'elle peine parfois à faire de la représentation une maîtrise et une rationalisation de ce qui, dans le réel, la déborde à l'infini. D’un côté, donc, la mosaïque de détails «montés » par l'écriture; de l'autre, l'élan qui tend vers l'infini du monde, dont le détail est sans doute l'expression vertigineuse, dans sa tentative toujours renouvelée pour l'épuiser et le défendre.

Cette force centrifuge, si caractéristique du génie romanesque d'Aragon, la phrase dilatoire de La Semaine sainte, qui s'enfle pour embrasser la profusion du passé et la vibration infinie du réel, la porte en elle. Le roman de 1958 rachète l'échec du roman médiéval, dont la phrase manifestait, elle aussi, la volonté de tout embrasser du passé, quitte à se distendre démesurément:

Toute la plaine qui est sur la rive gauche du fleuve grouillait de gens du roi. Les bûcherons dans la guerre de l'été précoce, nus jusqu'à la ceinture, mais portant

11. Louis Aragon, « Du décor» (1918), dans Chroniques, Paris, Stock, 1997, p. 25.

12. Michel Charles, «Le sens du détail », Poétique, n 116, novembre 1998. 
bonnet, achevaient de déblayer l'espace nécessaire à l'établissement du camp royal. Des arbres tombaient ici et là dans deux sens et on attelait des bœufs à les tirer vers le rivage. Déjà les tentes se montaient au milieu des jonchées de toiles et de piquets, et les enfants blonds du pays rôdant au milieu de la bousculade de mulets, de chevaux, de charrettes, regardaient d'yeux émerveillés le mélange d'hommes disparates qui fondaient cette Babylone provisoire, desquels il en était de si bruns que c'était épouvante, envoyés par le comte de Flandres et des Sarrazins d'Espagne, et des Anglais tachés de roux et brûlés de plaies roses pelantes, et des vilains amenés de Paris et de Bourgogne, petits, râblés, rapides à enfoncer les pieux et pendre les cordes. ${ }^{13}$

13. Louis Aragon, « Il y avait eu de grands signes dans le ciel », dans CEuvres romanesques complètes, Paris, Gallimard (Bibliothèque de la Pléiade), tome III, 2003, p. 1309. 